\title{
Single Dose of Pseudoephedrine Induces Simultaneous Bilateral Acute Angle Closure Crisis
}

\author{
Benjamin G. Spencer ${ }^{a} \quad$ Jonathan Baskin $^{b} \quad$ Blake F. Giarola ${ }^{b}$ \\ Jamie E. Craig ${ }^{c}$ \\ aTraining Medical Officer Unit, Flinders Medical Centre, Adelaide, SA, Australia; \\ ${ }^{b}$ Neurology Department, Royal Prince Alfred Hospital, Sydney, NSW, Australia; \\ 'Ophthalmology Department, Flinders Medical Centre, Adelaide, SA, Australia
}

\section{Keywords}

Angle closure $\cdot$ Glaucoma $\cdot$ Pseudoephedrine $\cdot$ Decongestants

\begin{abstract}
Simultaneous bilateral acute angle closure crisis (AACC) is a sight-threatening ocular emergency. Many "cold and flu" preparations contain compounds with sympathomimetic or anticholinergic qualities that confer a risk of inducing AACC. We present a review of cold and flu preparation-induced AACC, and present a case of simultaneous bilateral AACC triggered by a single oral dose of pseudoephedrine. The challenges facing the clinician in recognizing simultaneous bilateral AACC in the context of an upper respiratory tract infection are addressed. An awareness of this uncommon clinical entity, its pertinent clinical features, risk factors, and the drug classes that may precipitate an attack is critical for the timely diagnosis and management of this ocular emergency. Notably, clinicians must be aware that even a single dose of an implicated medication may trigger an attack of AACC.




\section{Case Reports in Ophthalmology}

\section{Introduction}

Acute angle closure crisis (AACC) is a sight-threatening ocular emergency. It typically presents as acute unilateral ocular/periocular pain and headache with rapidly progressive visual loss, sometimes associated with nausea and vomiting [1]. Examination of the affected eye typically reveals a mid-dilated unreactive or sluggish pupil, ciliary injection, a shallow anterior chamber, markedly reduced visual acuity (6/60 to hand motion), markedly raised intraocular pressure (above $50 \mathrm{~mm} \mathrm{Hg}$ ), and corneal haze due to corneal oedema [1]. AACC occurs due to occlusion of the trabecular meshwork by the peripheral iris, thus preventing outflow of aqueous humour from the anterior chamber of the eye [1]. This occlusion may be precipitated by a medication (typically a mydriatic) in an individual with ocular anatomy that predisposes them to AACC. Anatomical risk factors include having a small anterior segment (commonly seen in hypermetropes) and a crowded anterior segment which is usually due to an enlarged crystalline lens through normal ageing or cataract [2]. AACC is more prevalent in women and in East Asian and Inuit populations relative to Caucasian populations [1-3].

AACC can be triggered by numerous drug classes. Mydriasis is the mechanism of closure for adrenergic agonists, anticholinergics, and drugs with anticholinergic side effects [4]. Cholinergic agents may also induce AACC through anterior movement of the lens-iris diaphragm [4]. Sulfa-based drugs can induce AACC by causing ciliary body oedema and anterior displacement of the lens-iris diaphragm [4]. Cabergoline, a potent dopamine receptor agonist, has been implicated in one case of bilateral AACC with ciliary body effusion and anterior rotation postulated as possible mechanisms [5]. Oral, inhaled, intranasal, and topical preparations of the aforementioned classes may all induce AACC [6].

Our literature review revealed that numerous remedies for "cold and flu" symptoms have been implicated in triggering AACC. Cold and flu remedies often contain sympathomimetics aimed at causing vasoconstriction of blood vessels in the nasal mucosa to reduce secretions and congestion [7], and antihistamines (with anticholinergic side effects) aimed at blocking histamine release and subsequent coryzal symptoms [8]. In a case series of 72 patients presenting with AACC to a hospital in Hong Kong, 6 of these patients had taken "antitussive" agents which may have contained anticholinergic components [3]. A case of unilateral AACC was reported in a middle-aged man triggered by a cold preparation containing dextromethorphan (an opiate), promethazine (an antihistamine with anticholinergic side effects), and phenylpropanolamine (a sympathomimetic) [9]. Four cases of simultaneous bilateral AACC precipitated by cold and flu medications are reported in the medical literature. One case involved a middle-aged man who took over-the-counter nasal drops containing the adrenergic sympathomimetics, phenylephrine and naphazoline [10]. Another case involved a middleaged woman who took a preparation containing Atropa belladonna, a herb with anticholinergic properties [2]. The third case involved a middle-aged man who took tablets containing the $\alpha-1$ adrenergic receptor agonist, phenylephrine, and an antihistamine with anticholinergic properties, pheniramine maleate [11]. The fourth case was precipitated by a 5-day course of oral pseudoephedrine, a selective $\alpha-1$ adrenergic receptor agonist [12].

We present a case of simultaneous bilateral AACC in a middle-aged woman triggered by the use of over-the-counter cold and flu preparation, Mucinex $\mathrm{D}^{\circledR}$, containing pseudoephedrine hydrochloride $60 \mathrm{mg}$ and an expectorant, guaifenesin $600 \mathrm{mg}$. To our knowledge, this is the second published case of bilateral AACC induced by oral pseudoephedrine, and the first reported case of AACC precipitated by a single dose of a cold and flu remedy. 


\section{Case Report}

A 59-year-old lady presented to a tertiary hospital emergency department with a 2-day history of blurred vision and headache in the context of an upper respiratory tract infection. She had taken a single tablet of "Mucinex D" prior to the onset of her visual disturbance. She denied any ocular trauma and had no significant ophthalmic history. Her medical history was only significant for hypothyroidism for which she took thyroxine as her only regular medication. On examination, her visual acuity (pinhole) was 6/60+2 on the right and hand motion on the left. The conjunctivae were injected bilaterally, and both pupils were mid-dilated and unreactive to light. The patient was referred to the neurology service for further assessment. The neurology service promptly referred the patient to the ophthalmology service given concerns of possible AACC.

Further ophthalmic assessment revealed bilaterally oedematous corneas, shallow anterior chambers, and intraocular pressures of 53 and $51 \mathrm{~mm} \mathrm{Hg}$ on the right and left, respectively. A diagnosis of simultaneous bilateral AACC was made. She received acetazolamide 500 mg i.v. in addition to brinzolamide, brimonidine, latanoprost, timolol, and pilocarpine drops. She then underwent bilateral YAG peripheral iridotomy with argon-pre-treatment. Post-procedure pressures were 9 and $32 \mathrm{~mm} \mathrm{Hg}$ on the right and left, respectively. She was commenced on dexamethasone $0.1 \%$ topically bilaterally q.i.d., travoprost topically in the left eye only, and continued on the timolol and brinzolamide topically in the left eye only. The following day her visual acuity (pinhole) was $6 / 9+1$ and $6 / 15$ in the right and left eyes, respectively. Her pressures were 7 and $8 \mathrm{~mm} \mathrm{Hg}$, respectively.

\section{Discussion/Conclusion}

Simultaneous bilateral AACC is an uncommon but sight-threatening condition for which clinicians must be highly vigilant. This is particularly the case in patients presenting with symptoms in the context of an upper respiratory tract infection for which a decongestant preparation has been used by the patient. The symptomology of simultaneous bilateral AACC, including headache, ocular pain, and red eye, may overlap with the flu-like symptoms for which the medications that might trigger AACC are taken. Furthermore, blurred vision is a well-recognised side effect of anticholinergic and sympathomimetic decongestants, and thus this symptom may not alert the patient or clinician to the seriousness of the situation. The simultaneous bilateral onset of visual disturbance may erroneously lead a clinician away from suspecting an ophthalmic process in favour of a central neurological pathology, as initially occurred in this case. As was also illustrated by this case, obtaining a complete drug history is imperative, as even a single dose of an over-the-counter preparation can precipitate AACC. The progressive nature of symptoms after taking an implicated medication should prompt the clinician to consider simultaneous bilateral AACC as a possible diagnosis. Urgent ophthalmological assessment is indicated in suspected cases.

\section{Statement of Ethics}

This case complies with the tenets of the Declaration of Helsinki. The patient gave written informed consent for the publication of this case report. 


\section{Disclosure Statement}

The authors have no conflicts of interest to declare.

\section{Funding Sources}

No funding was received or utilised in relation to the preparation of this case report.

\section{Author Contributions}

Benjamin J. Spencer: drafting of the manuscript, patient consenting, and literature review. Jonathan Baskin: patient assessment and manuscript review. Blake F. Giarola: patient assessment, data collection, and manuscript review. Jamie E. Craig: review of the manuscript, provision of specialist knowledge in analysis of the case, and interpretation of significance in the context of the literature review.

\section{References}

1 Ah-Kee EY, Egong E, Shafi A, Lim LT, Yim JL. A review of drug-induced acute angle closure glaucoma for nonophthalmologists. Qatar Med J. 2015 May;2015(1):6.

2 Rudkin AK, Gray TL, Awadalla M, Craig JE. Bilateral simultaneous acute angle closure glaucoma precipitated by non-prescription cold and flu medication. Emerg Med Australas. 2010 Oct;22(5):477-9.

3 Lai JS, Liu DT, Tham CC, Li RT, Lam DS. Epidemiology of acute primary angle-closure glaucoma in the Hong Kong Chinese population: prospective study. Hong Kong Med J. 2001 Jun;7(2):118-23.

4 Tripathi RC, Tripathi BJ, Haggerty C. Drug-induced glaucomas: mechanism and management. Drug Saf. 2003;26(11):749-67.

5 Razmjoo H, Rezaei L, Dehghani A, Peyman A, Akhlaghi M. Bilateral angle-closure glaucoma in a young female receiving cabergoline: a case report. Case Rep Ophthalmol. 2011 Jan;2(1):30-3.

6 Lai JS, Gangwani RA. Medication-induced acute angle closure attack. Hong Kong Med J. 2012 Apr;18(2):13945.

7 Johnson DA, Hricik JG. The pharmacology of alpha-adrenergic decongestants. Pharmacotherapy. 1993 NovDec;13(6 Pt 2):110S-5S.

8 De Sutter AI, Lemiengre M. Antihistamines for the common cold. Cochrane Database Syst Rev [Internet]. 2003. Available from: https://doi.org/10.1002/14651858.CD001267.

9 Barrett V, Jordan T. Angle closure risk from proprietary medicines. Eye (Lond). 2001 Apr;15(Pt 2):248-9.

10 Khan MA, Watt LL, Hugkulstone CE. Bilateral acute angle-closure glaucoma after use of Fenox nasal drops. Eye (Lond). 2002 Sep;16(5):662-3.

11 Nicoară SD, Damian I. Bilateral simultaneous acute angle closure attack triggered by an over-the-counter flu medication. Int Ophthalmol. 2018 Aug;38(4):1775-8.

12 Ah-Kee EY, Li Yim JF. Bilateral acute angle closure glaucoma precipitated by over the counter oral decongestant. Int J Ophthalmol. 2014 Apr;7(2):387-8. 